\title{
X-RAY ASTRONOMY SATELLITE GINGA
}

\author{
F. MAKINO \\ Institute of Space and Astronautical Science, 3-1-1, Yoshinodai, \\ Sagamihara, Kanagawa 229, Japan.
}

\begin{abstract}
The X-ray astronomy satellite Ginga carries three scientific instruments, the Large Area proportional Counters (LAC), All Sky X-ray Monitor (ASM) and Gamma-ray Burst Detector (GBD). The LAC is the main instrument with an effective area of 4000 $\mathrm{cm}^{2}$ giving it the highest sensitivity to hard X-rays so far achieved. Ginga observed about 250 targets up to the end of 1989.
\end{abstract}

\section{Introduction}

Ginga is the third Japanese X-ray astronomy satellite following Hakucho (Kondo et al. 1981) and Tenma (Tanaka et al. 1984). The fabrication, launching and operation of Ginga were carried out entirely by the Institute of Space and Astronautical Science (ISAS). General properties of Ginga have been described in the literature (Makino et al. 1987) and are summarized in Table 1. Ginga was launched on February 5, 1987 from Kagoshima Space Center of ISAS by the M-3S-II launch vehicle. The initial orbital parameters were a perigee height of $505.5 \mathrm{~km}$, an apogee height of $673.5 \mathrm{~km}$, an inclination of $31.1^{\circ}$ and a period of $96.5 \mathrm{~min}$. The altitude of Ginga has decreased rapidly since early 1989 , because of high solar activity. The perigee and apogee height were $575.1 \mathrm{~km}$ and $471.0 \mathrm{~km}$ respectively in April 1990 .

The total mass of the satellite is $420 \mathrm{~kg}$ of which $105 \mathrm{~kg}$ is for scientific instruments, and the rectangular main body measures $1 \mathrm{~m} \times 1 \mathrm{~m} \times 1.5 \mathrm{~m}$ with four deployable solar panels $1.7 \mathrm{~m}$ long and $0.75 \mathrm{~m}$ wide. A maximum power of $500 \mathrm{~W}$ can be generated from the solar panels and normal power consumption is about $150 \mathrm{~W}$.

The scientific instruments are the Large Area proportional Counters (LAC), All Sky X-ray Monitor (ASM) and Gamma-ray Burst Detector (GBD). The LAC and GBD are prepared in collaboration with University of Leicester and Rutherford/Appelton Laboratory in the U.K. and Los Alamos National Laboratory in the U.S.A. respectively.

All the instruments have functioned normally to date, April 1990. The total number of targets observed to the end of 1989 is about $250,51 \%$ are Galactic sources and $49 \%$ extra-Galactic sources.

\section{Scientific instruments}

\subsection{Large Area proportional Counters (LAC)}

The LAC is the primary instrument of Ginga and consists of eight identical counters (Turner et al., 1989). The total effective area is $4000 \mathrm{~cm}^{2}$. Each counter is a multicell proportional counter which comprises 13 cell counters of four layers. The cell

Y. Kondo (ed.), Observatories in Earth Orbit and Beyond, 41-48.

(C) 1990 Kluwer Academic Publishers. Printed in The Netherlands. 


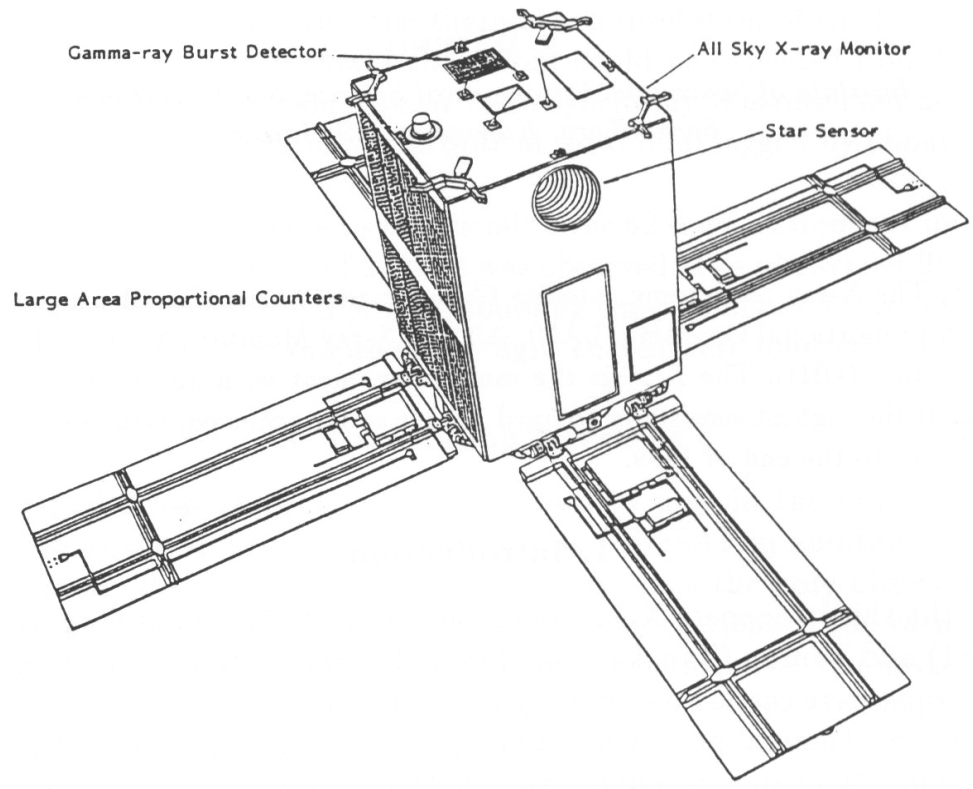

Fig. 1. The outside view of Ginga.

\section{TABLE I}

General characteristics of Ginga

Launch

Launch Vihicle

Initial Orbit

Mass

Experiments

Stabilization

Attitude Sensors

Telemetry Bit Rate Data Recorder
February 5, 1987

M-3SII-3

Perigee : $505.5 \mathrm{~km}$, Apogee : $673.5 \mathrm{~km}$

Inclination : $31.1^{\circ}$, Period : $96.5 \mathrm{~min}$

Total : $420 \mathrm{~kg}$, Experiments : $105 \mathrm{~kg}$

Large Area Proportional Counters (LAC)

All Sky X-ray Monitor (ASM)

Gamm-ray Burst Detector (GBD)

Biased momentum three-axis control

Two CCD Star Trackers, Sun Sensor, Four Gyroscopes and Geomagnetic Aspectmeters

High:16384 bps, Medium:2048 bps, Low:512 bps 41.9 Mbit Bubble Memory

Recording Time:42m40s(H), 5h41m(M), 22h44m(L) 
counters sided by counter wall are connected together and used for anti-coincidence to lessen background, as well as the signals from the end plates. Because most of the background counts are produced by Compton scattering of hard X-rays and gammarays in the counter body. The anodes of the central two layers are connected together and those of the cell counters sided by entrance window are connected alternately. The signals from these three anode groups are analyzed by onboard data processor and are transmitted. Mutual anti-coincidence among these three anode groups is also employed for background reduction.

The entrance window is beryllium foil of $62 \mu \mathrm{m}$ thick which is supported by honeycomb type collimators made with stainless steel plates. The counters are filled with a gas mixture of argon (75\%), xenon (20\%) and carbon dixide (5\%) at 2 atm at $293 \mathrm{~K}$. The sensitive energy range is from $1.5 \mathrm{keV}$ to $35 \mathrm{keV}$. The collimator field of view is elliptical of 1 degrees by two degrees (FWHM). The counting rate of X-rays from Crab Nebula is about $10000 \mathrm{cps}$, while the background rate is $70 \mathrm{cps}$ of which $18 \mathrm{cps}$ is due to cosmic diffuse X-ray background (CDXB). The detection limit of the LAC is confusion limit which is defined as sky to sky fluctuation of CDXB due to intensity source number relation. The $3 \sigma$ limit is $2.1 \mathrm{cps}$, roughly equal to $0.2 \mathrm{mCrab}$ (Hayashida et al. 1989). The sensitivity is the highest so far achieved in this energy region.

The time resolution depends on the number of energy channels for pulse height analysis and the telemetry bit rate. The highest is $1 \mathrm{~ms}$ in the case of 2 energy channels and $16 \mathrm{kbps}$, and the lowest is $16 \mathrm{~s}$ in the case of 48 energy channel and 500 bps. However, a temperature dependence of the clock frequency appeared after launch, but can be compensated for using temperature data for high resolution timing analysis (Deeter and Inoue, 1990). No degradation of the LAC has been observed to the end of April 1990.

\subsection{All Sky X-Ray Monitor (ASM)}

The ASM consists of two identical proportional counters, each containing three independent counters with veto cell counters at the back (Tsumeni et al. 1989). These six counters are attached to a collimator of $1^{\circ}$ by $45^{\circ}$ (FWHM) of various slant angles. The monitoring of a wide sky region is conducted by slow rotation of the satellite around the z-axis. The position of the source can be determined by the time deferences of the peak position of the source for each counter. The normal frequency of scanning is once per day. The sky region which can be monitored is constrained to the equatorial region of satellite coordinate system determined by the LAC target.

The effective area of each counter is $70 \mathrm{~cm}^{2}$. The counters are filled with xenon $(96.7 \%)$ and carbon dioxide $(3.3 \%)$ at 1 atm. The thickness of beryllium windows are $50 \mu \mathrm{m}$. The detectors are sensitive to X-rays from $1 \mathrm{kev}$ to $30 \mathrm{keV}$. The detection threshold is about $50 \mathrm{mCrab}$ but becomes higher with increasing elevation angle from X-y plane of the satellite. 


\subsection{Gamma-RAy burst DETeCtor (GBD)}

Two detectors, a $\mathrm{NaI}(\mathrm{Tl})$ scintillation counter and a Xe-filled proportional counter have been used for gamma-ray burst observation (Murakami et al. 1989). The $\mathrm{NaI}(\mathrm{Tl})$ is $8.8 \mathrm{~cm}$ in diameter and $1 \mathrm{~cm}$ in thickness, and measures hard X-rays from $13 \mathrm{keV}$ to $400 \mathrm{keV}$. The proportional counter has an effective area of $63 \mathrm{~cm}^{2}$, covering energy region from $2 \mathrm{keV}$ to $30 \mathrm{keV}$. The $\mathrm{X}$-ray observation of gamma-ray bursts has been conducted for the first time.

The gamma-ray bursts are unpredictable transient events shorter than 1 min. Two memory systems are employed to record bursts. The one is a fast memory triggered by the rise of the burst and is frozen until read out by command from the ground station. The sampling time is $31 \mathrm{~ms}$ for pulse counts and $0.5 \mathrm{~s}$ for pulse height distributions. The data in the time interval $16 \mathrm{~s}$ before and $48 \mathrm{~s}$ after the onset of the burst are recorded. The "time-to-spill" mode of data acquisition was used to cover wide dynamic range. The onset time are measured with accuracy of $244 \mu \mathrm{s}$. Only one burst can be stored in the fast memory. The other memory is a continuously sampling slow memory which sampling rate is either $125 \mathrm{~ms}, 1 \mathrm{~s}$ or $4 \mathrm{~s}$ depending on bit rate. The GBD includes small semiconductor detector to monitor radiation belt (RBM) and generate RBM flag to turn off high voltage supplies for LAC, ASM and GBD.

\section{Notable results obtained with Ginga}

\subsection{Cyclotron features of PUlsar spectra}

The merit of the LAC is high sensitivity in the energy region higher than $10 \mathrm{keV}$. Spectral structures in the hard X-ray region were detected from five X-ray pulsars, Her X-1 (Mihara et al. 1990), 4U 1538-52 (Clark et al. 1990), V 0332+53 (Makishima et al. 1990), 1E 2259+58 (Koyama et al. 1989a) and 4U 0115+634). The most pronounced spectrum was obtained from V0332+53 on October 1, 1989, as shown in Fig. 2. The spectrum can be expressed by power law with resonant absorption at $28.5 \mathrm{keV}$.

Possible interpretation of this structure is cyclotron absorption by electrons in highly magnetized plasma. The magnetic field can be estimated as $2.5 \times 10^{12} \mathrm{G}$. Further analysis, such as pulse phase dependence of absorption energy and depth will reveal X-ray transport in pulsar plasma.

\subsection{X-RAY SCATTERING BY INTERSTELLAR DUST GRAINS}

The moon occulted accidentally transient X-ray source (GS 1741-28) appeared near the Galactic center. The observation was conducted on October 26, 1987 (Mitsuda et al. 1990). The light curves in three energy bands are shown in Fig. 4. The gradually decreasing and increasing component around the point source, which corresponds to sharp fall and rise is clearly seen at ingress and egress respectively. The light curves show that this extended component decreases with increasing Xray energy. One can conclude that the extended component is due to scattering by cosmic dust grains from the energy dependence of relative intensity and angular 


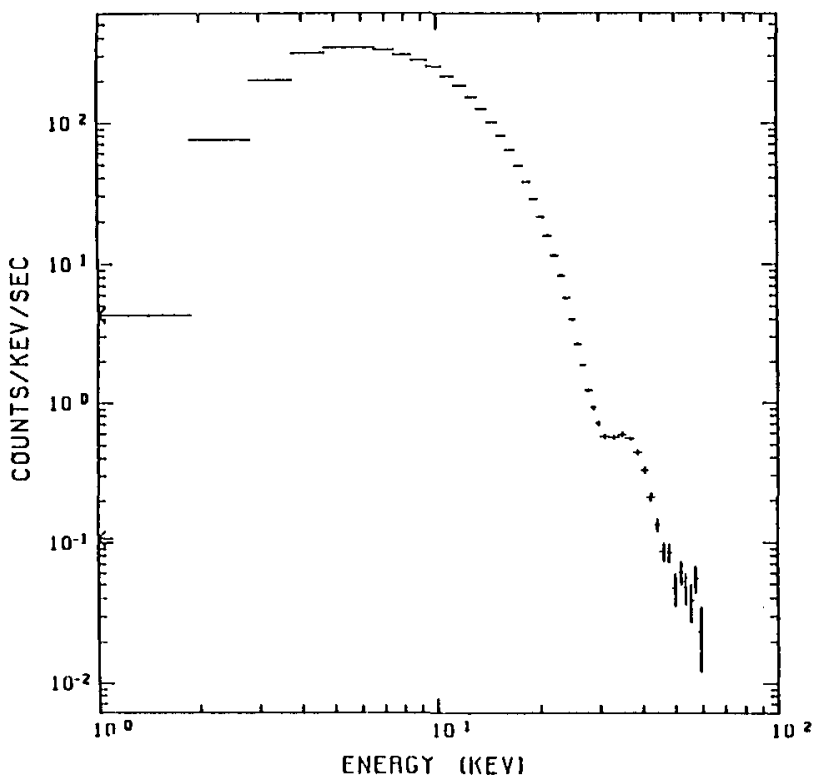

Fig. 2. The spectrum of X-ray transient pulsar V $0332+53$. An absorption feature at 28 $\mathrm{keV}$ is clearly seen (Makishima et al. 1990).

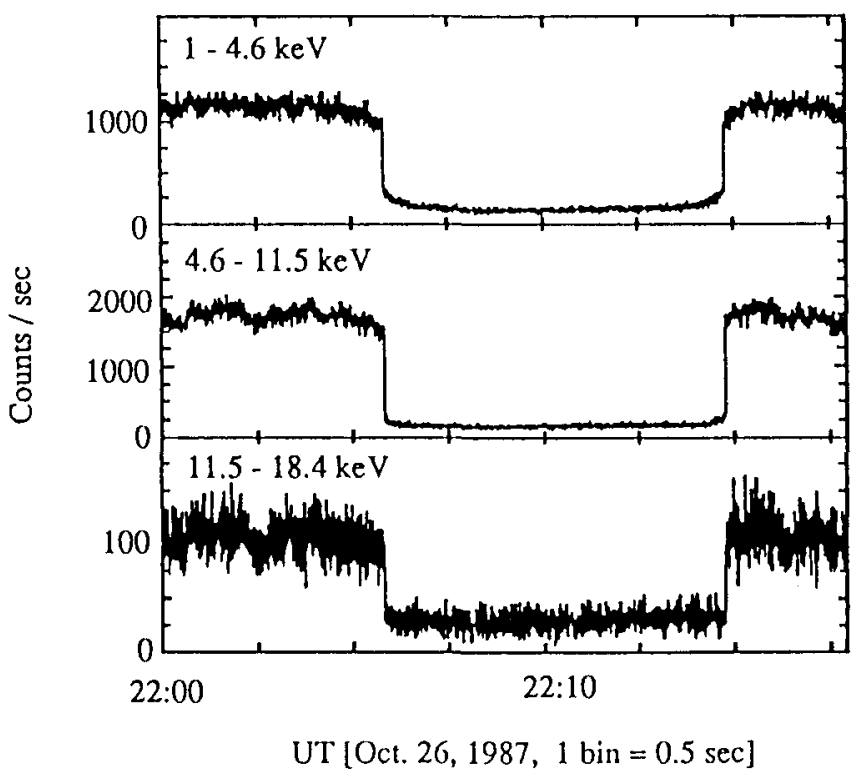

Fig. 3. Light curves of transient X-ray source GS $1741-28$ in a occasion of lunar occultation. The slowly varying component is clearly visible at ingress and egress (Mitsuda et al. 1990). 


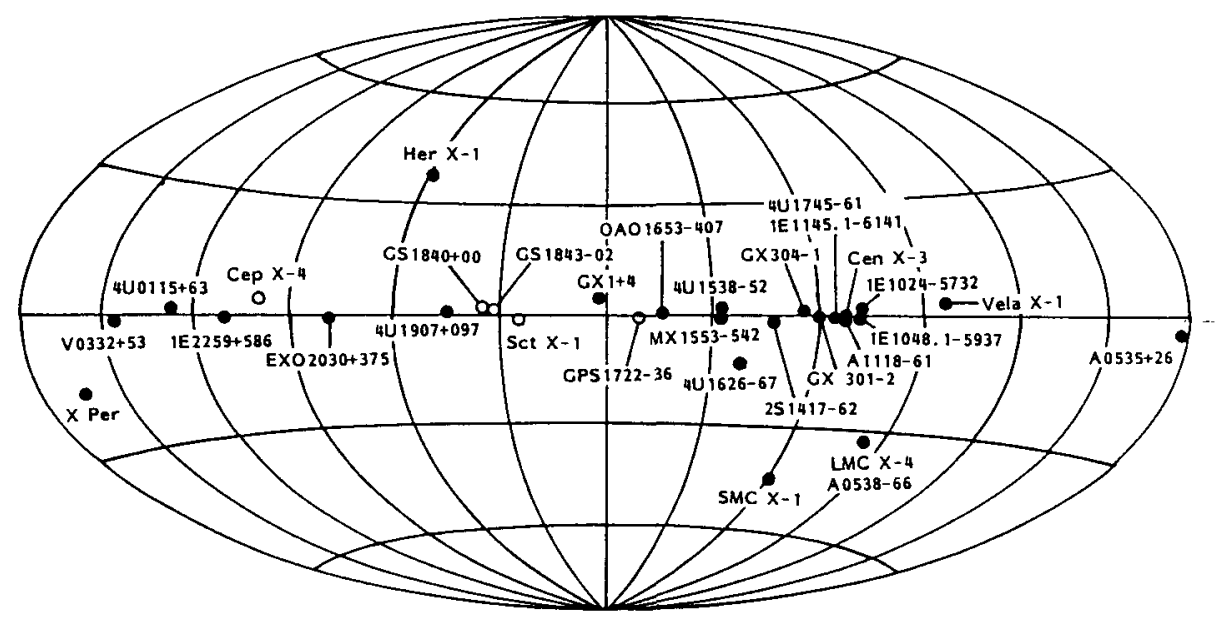

Fig. 4. Distribution of X-ray pulsars in Galactic coordinate. New pulsars discovered with Ginga are indicated by open circles.

diameter. The size and chemical composition responsible for X-ray scattering were estimated as about $0.1 \mu \mathrm{m}$ in diameter and iron compound.

\subsection{Discovery of neW transient X-Ray pulsars}

Five X-ray pulsars, Cep X-4, GS 1843+00, GPS 1722-362, GS 1843-02 and Sct $\mathrm{X}-1$ have been discovered with Ginga (Koyama and Takeuchi, 1989). The scanning observation of Galactic plane discovered several transient X-ray sources. Most of new sources are faint and have the hard X-ray spectra characteristic of X-ray pulsars. The low energy part of the spectra is heavily absorbed by interstellar matter, and hydrogen column densities are higher than $10^{23} \mathrm{H}$-atoms $\mathrm{cm}^{-2}$. These new transients which are candidate pulsars and new pulsars are concentrated in Scutum region, possibly located in $5 \mathrm{kpc}$ Galactic ring. The total number of X-ray pulsars known before Ginga is 26 and they are located within a few kpc from solar system. Ginga observations suggest existence of many faint pulsars associated with inner Galactic arms.

\subsection{INTENSE IRON LINE EMISSION FROM GALACTIC CENTER}

The scanning observation along Galactic plane revealed diffuse emission of iron line peaked at Galactic center (Koyama et al. 1989b). The line energy of $6.7 \mathrm{keV}$ suggests the emission is from high temperature plasma. The distribution of iron line shows sharp peak at Galactic center with angular diameter of $1.8^{\circ}$ corresponding to $300 \mathrm{pc}$ at the Galactic center. The peak flux is $1.5 \times 10^{-7} \mathrm{erg} \mathrm{s}^{-1} \mathrm{sr}^{-1} \mathrm{~cm}^{-2}$ and integrated luminosity of iron line is $2.3 \times 10^{36} \mathrm{erg} \mathrm{s}^{-1}$. The distribution of iron line extends to Galactic ridge. A possible origin of high temperature plasma 


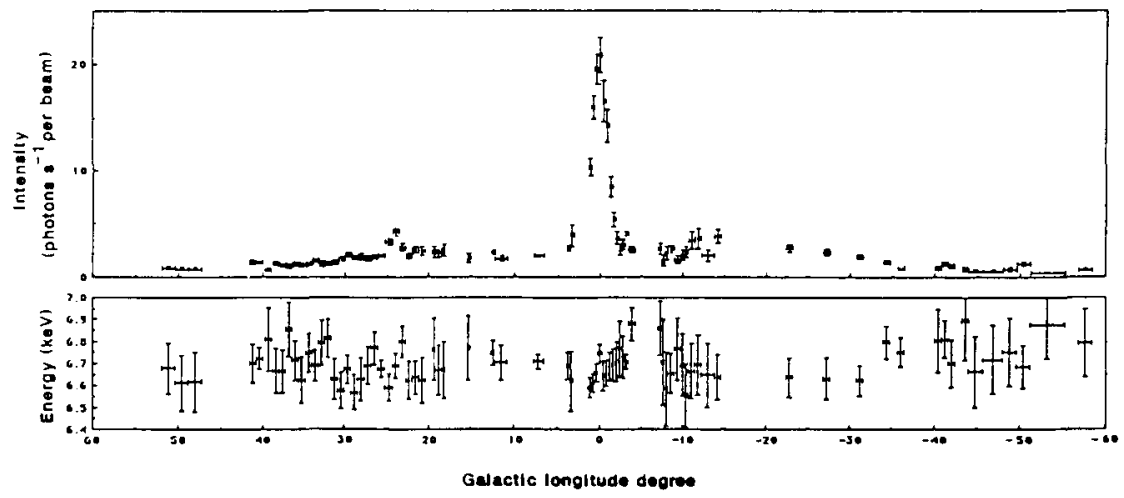

Fig. 5. Galactic longitude distribution of iron K-line. Lower panel shows line energy (Koyama et al. 1989b).

is supernova remnants. The distribution in such a narrow region suggests that the succesive explosion occurred at the epoch not earlier than 105 years.

\subsection{Spectral features and variability of Seyfert galaxies}

Important discoveries were made on X-ray emission from Seyfert galaxies by Ginga. The iron line emission and $\mathbf{K}$-absorption edge were observed from all Seyfert galaxies observed with Ginga (Pounds, 1989, Matsuoka et al., 1990, Kunieda et al., 1990 ). The line energy is $6.4 \mathrm{keV}$ that is fluorescent $\mathrm{X}$-rays from cold matter. The equivalent widths of iron line were about $100 \mathrm{eV}$ or more. While the absorbing matter density determined from spectra below $2 \mathrm{keV}$ is less than $10^{22} \mathrm{H}$-atom $\mathrm{cm}^{-2}$. Therefore, the reprocessing matter is not distribute along the line of sight but it should sustend wide solid angle to central source. A possible configuration is an accretion disc (George et al. 1989). The variability of iron line emission from the low luminosity Seyfert 1 galaxy, NGC 6814 was observed by Kunieda et al. (1990). They obtained a positive correlative variation between the iron line and the continuum, and time lag between them was shorter than $250 \mathrm{~s}$. This is comparable to observed variability time scale. This suggests that a geometrically thin cold accretion disc extends to the vicinity of central source. On the other hand, the continuum emission from NGC 4051 and MCG-6-30-15 varied in correlated way with spectral indices (Matsuoka et al. 1990). The spectra became steeper with increasing flux. This may be a further evidence for the existence of a scattered continuum which variability time scale is longer than the main component. Ginga observed Seyfert 2 galaxies, NGC 1068 (Koyama et al. 1989c), Mkn 348 (Warwick et al. 1989) and Mkn 3 (Awaki et al. 1990). Intense iron line emission at $6.4 \mathrm{keV}$ was observed from NGC 1068 and Mkn 3. The equivalent widths were as high as $1 \mathrm{keV}$. The absorbing matter densities were in the order of $10^{23} \mathrm{H}$-atom $\mathrm{cm}^{-2}$ for $\mathrm{Mkn} 348$ and Mkn 3. These facts support an idea that the Seyfert 2 galaxies are obscured Seyfert 1 galaxies. This may have an impact on the origin of the cosmic diffuse 
X-ray background.

\section{Concluding remark}

Ginga has achieved highest sensitivity in the energy range from $1.5 \mathrm{keV}$ to $40 \mathrm{keV}$ and has revealed new aspects of X-ray astronomy.

\section{Acknowledgements}

Author would like to thank all the members of Ginga team for their preparation and operation of Ginga and for providing him with results of the observations. $\mathrm{He}$ acknowledge Dr. C. Day for careful reading of the manuscript and for his comments.

\section{References}

Awaki, H., et al: 1990, Publ. Astron. Soc. Japan, submitted

Clark, G. W., et al: 1990, Astrophys.J. 353, 274

Deeter, J. E., and Inoue, H.: 1990, ISAS Research Note No. 430,

George, I. M., et al: 1989, ESA SP-296 2, 945

Hayashida, K., et al: 1989, Publ. Astron. Soc. Japan 41, 373

Kondo, I., et al: 1981, Space Sci. Instr. 5, 211

Koyama, K., and Takeuchi, Y.: 1989, ESA SP-296 1, 483

Koyama, K., et al: 1989a, Publ. Astron. Soc. Japan 41, 461

Koyama, K., et al: 1989b, Nature 339, 603

Koyama, K., et al: 1989c, Publ. Astron. Soc. Japan 41, 731

Kunieda, H., et al:: 1990, Nature, in press

Makino, F., et al: 1987, Astrophys. Lett. and Communication 25, 223

Makishima, K., et al: 1990, in preparation

Matsuoka, M., et al.: 1990, Astrophys. J., in press

Mihara, T., et al.: 1990, Nature, submitted

Mitsuda, K., et al: 1990, Astrophys. J. 353, 480

Murakami, T., et al: 1989, Publ. Astron. Soc. Japan 41, 405

Ninomiya, K., et al: 1984, Proc. of IFAC meeting, 2915

Pounds, K. A.: 1989, ESA SP-296 2, 753

Tanaka, Y., et al: 1984, Publ. Astron. Soc. Japan 36, 641

Tsunemi, H., et al: 1989, Publ. Astron. Soc. Japan 41, 391

Turner, M. J. L., et al: 1989, Publ. Astron. Soc. Japan 41, 345

Warwick, R. S., et al: 1989, Publ. Astron. Soc. Japan 41, 739 